\title{
冰期快速形成导致奥陶纪末生物大灭绝
}

\author{
孙卫东 $1,2,3^{*}$, 廖仁强 1,3
}

1. 中国科学院海洋研究所, 中国科学院海洋大科学中心, 深海中心, 青岛 266071;

2. 青岛海洋科学与技术试点国家实验室, 海洋矿产资源评价与探测技术功能实验室, 青岛 266237;

3. 中国科学院大学, 北京 100049

* 联系人, E-mail: weidongsun@qdio.ac.cn

\section{The end Ordovician mass extinction induced by rapid glaciation}

\author{
Weidong $\operatorname{Sun}^{1,2,3^{*}} \&$ Renqiang Liao ${ }^{1,3}$ \\ ${ }^{1}$ Center of Deep Sea Research, Center for Ocean Mega-Science, Institute of Oceanology, Chinese Academy of Sciences, Qingdao 266071, China; \\ ${ }^{2}$ Laboratory for Marine Mineral Resources, Pilot National Laboratory for Marine Science and Technology (Qingdao), Qingdao 266237, China; \\ ${ }^{3}$ University of Chinese Academy of Sciences, Beijing 100049, China \\ * Corresponding author, E-mail: weidongsun@qdio.ac.cn
}

doi: 10.1360/TB-2019-0773

在显生宙以来, 地球上发生了 5 次生物大灭绝事件. 其中, 奥陶纪末生物大灭绝(the end Ordovician mass extinction, EOME) 是第一次全球生物大灭绝, 其生态系统遭 受的破坏程度排在 5 次生物大灭绝事件的第二位 ${ }^{[1 \sim 3]}$. 绝大 多数生物大灭绝事件都与大火成岩省在时间上密切相 关 ${ }^{[4]}$, 但是奥陶纪末生物大灭绝的原因至今仍存在争论. 在奥陶纪-志留纪界线, 曾经发生过一次大规模冰川事件. 一些学者认为冰川导致的剧烈气候变迁是造成这次生物 大灭绝的关键 ${ }^{[5]}$.

值得注意的是, 在国际地层表中(International Chronostratigraphic Chart, ICC), 奥陶纪-志留纪界线的年龄为 $443.8 \pm 1.5 \mathrm{Ma}$, 不是高精度年龄, 而是根据精度相对较低 的多颗粒锆石 $\mathrm{U}-\mathrm{Pb}$ 等定年结果估算的. 这种精度的年龄不 足以讨论奥陶纪末生物大灭绝的持续时间等重要科学问 题, 制约了对这一问题的理解.

华南有全球保存最完好的奥陶系-志留系界线地层. 著名的赫南特阶(Hirnantian)金钉子就选在三峡地区. 在奥 陶系-志留系界线附近, 华南广泛发育多层钾质斑脱岩. 这 种岩石通常是火山灰在碳酸盐台地上发生蚀变形成的. 斑 脱岩里面的锆石是测定地层界线年龄的理想样品. 前人利 用离子探针测定过三峡地区斑脱岩中的锆石 $\mathrm{U}-\mathrm{Pb}$ 年龄, 获 得了赫南特阶的年龄为 $443.3 \pm 1.6 \mathrm{Ma}^{[6]}$, 与 ICC给出的奥陶 纪-志留纪界线年龄一致. 但是由于离子探针获得的年龄 数据精度一般在 $1 \%$, 不是确定地层界线的理想方法.

2019年12月, Solid Earth Sciences发表了中国科学院 南京地质古生物研究所詹仁斌和中国科学院广州地球化
学研究所凌明星团队与澳大利亚国立大学 ${ }^{[7]}$ 合作的研究成 果, 首次给出了奥陶系-志留系界线附近多个层位的高精 度锆石U-Pb年龄.

上述研究团队在云南东南部的万和村发现了一个保 存完好的奥陶纪-志留纪界线层位(图1). 这个层位中的上奥 陶统大渡河组与扬子地台的五峰组对应. 在此层位之上为 上奥陶统赫南特阶观音桥层和下志留统龙马溪组. 在此奥 陶系-志留系过渡层位中, 有十几层的钾质斑脱岩夹在薄层 灰岩间, 厚度从毫米级到厘米级. 该研究团队对上述地层 剖面进行了详细的工作, 采集了数百千克的斑脱岩样品, 挑选锆石, 在澳大利亚国立大学地球科学研究院同位素实 验室进行了双稀释剂高精度热电离质谱(ID-TIMS)单颗粒锆 石 $\mathrm{U}-\mathrm{Pb}$ 定年，获得了 4 个高精度的同位素年龄，包括： 444.65 $\pm 0.22 \mathrm{Ma}$ (middle Dicellograptus complexus Biozone), $444.06 \pm 0.20 \mathrm{Ma}$ (lower Paraorthograptus pacificus Biozone), $443.81 \pm 0.24 \mathrm{Ma}$ (upper Tangyagraptus typicus Subzone)和 442.99 $\pm 0.17 \mathrm{Ma}$ (upper Metabolograptus extraordinarius Biozone). 并以此为基础, 计算出奥陶纪-志留纪界线年龄 $(442.67 \pm 0.24 \mathrm{Ma})$, 赫南特阶底界 $(443.14 \pm 0.24 \mathrm{Ma}) 、 D$. mirus Subzone底界(443.41 $\pm 0.24 \mathrm{Ma}) 、 T$. typicus Subzone底 界(444.17 $\pm 0.28 \mathrm{Ma}) 、$ P. pacificus Biozone底界(444.38 \pm 0.31 $\mathrm{Ma})$ 和 $D$. complexus Biozone底界 $(444.84 \pm 0.31 \mathrm{Ma})$ 年龄. 这 些结果显示, 赫南特阶顶界和底界年龄明显小于国际地层 年代表 (ICC)中给出的年龄, 赫南特阶持续时间为 $0.47 \pm$ $0.34 \mathrm{Ma}$, 远远短于以前的推测 ${ }^{[7]}$. 由此, Ling等人 ${ }^{[7]}$ 推断冰 川快速形成过程导致的剧烈气候变迁可能是奥陶 


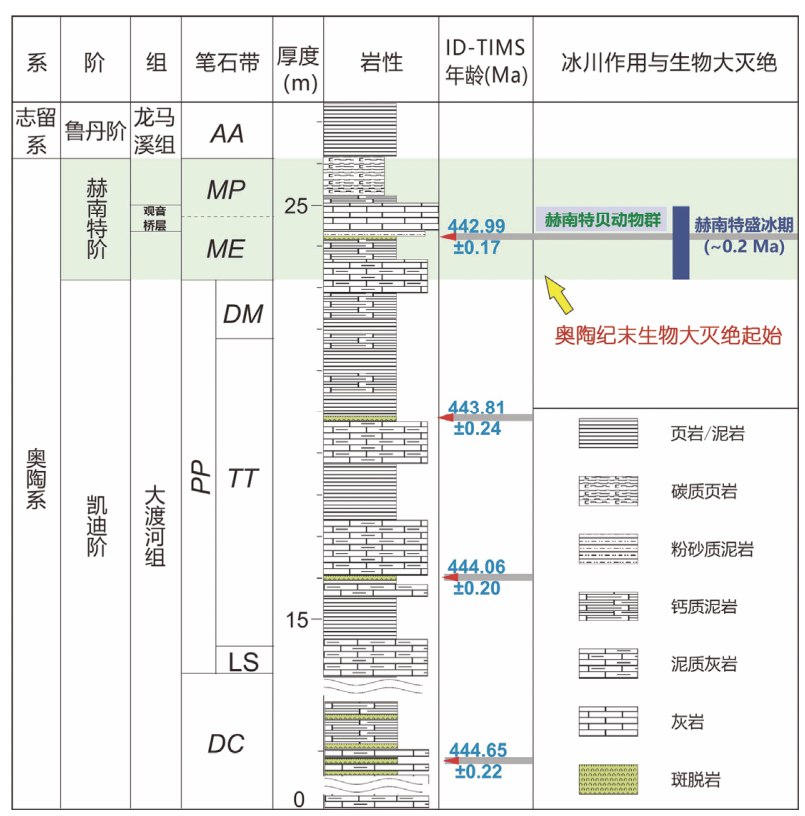

图 1 (网络版彩色)云南万和奥陶系-志留系剖面斑脱岩高精度U-Pb 定年及其对奥陶纪末生物大灭绝的指示. 修改自文献[7]

Figure 1 (Color online) High precision U-Pb dating of bentonite in the Wanhe section, Yunnan Province, with indication of the end Ordovician mass extinction (EOME). Modified from Ref. [7]

纪末生物大灭绝的主要原因. 这一成果不仅是对国际地层 年代学的重要贡献, 也有助于揭示奥陶纪末生物大灭绝的 成因.

研究表明, 在冰川形成过程中, 由于海平面下降, 大 洋板块减压上浮, 促进软流圈地幔的减压部分熔融, 导致 大洋中脊玄武岩喷发加强 ${ }^{[8]}$. 与此相反, 在化冰过程中, 由于陆地冰川卸载、减压, 陆相火山活动通常会加强(图 $2)^{[9]}$. 华南地区的奥陶系-志留系界线附近地层广泛发育的 大规模火山岩沉积, 显示在冰期发育过程中火山活动加强 的趋势. 这可能是由于华南地区当时位于赤道附近, 且大 面积处于水下环境, 在全球大规模冰川发育过程中, 华南 大陆并未增加冰量, 相反其负载的海水变浅, 引起减压部 分熔融, 促进了火山活动.
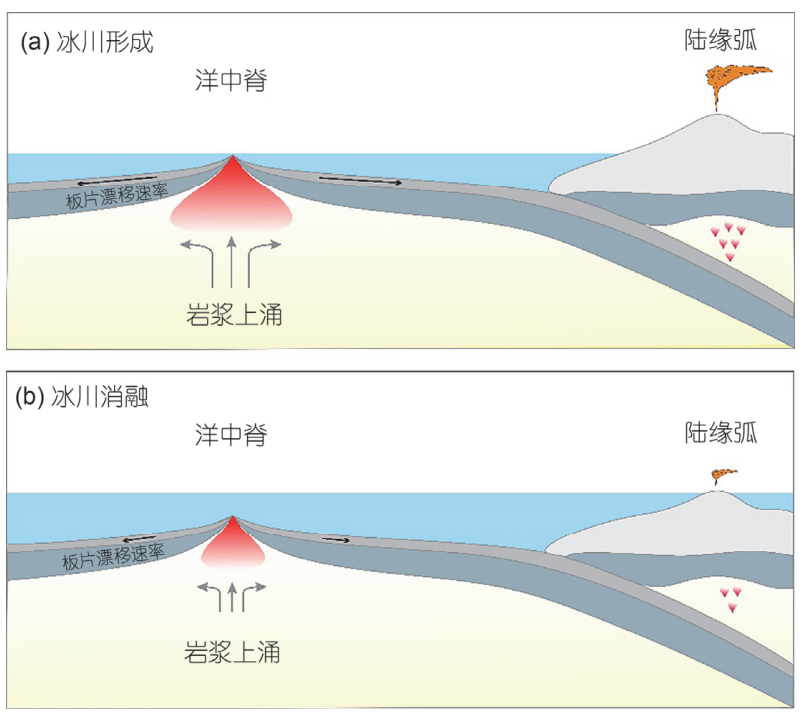

图 2 (网络版彩色) 冰川形成过程对洋中脊和俯冲带火山活动的促进 机理. 冰川形成过程中海平面下降促进大洋中脊玄武岩喷发, 导致 洋中脊受热程度加强, 洋中脊相对于老洋壳的高度增加, 板块倾斜 度增加, 进而导致板块扩张速度增加, 岛弧火山岩喷发加强

Figure 2 (Color online) The influence of glaciation on both mid-ocean ridge basalt (MORB) and arc volcanisms. During glaciation, the MORB volcanism is enhanced due to sea level drop. Meanwhile, the spreading ridge is more elevated compared to older oceanic crust, such that the drifting rate is increased, intensifying plate subduction and arc volcanisms

另一方面, 这些斑脱岩的地球化学组成显示陆缘弧 火山岩的特征, 说明华南周缘在奥陶纪-志留纪时期可能 存在岛弧 ${ }^{[10]}$. 海平面下降在导致大洋中脊玄武岩喷发加强 的同时, 会导致洋中脊相对于老洋壳抬升、大洋板块倾斜 角度增加, 从而提高大洋板块俯冲的速度 ${ }^{[11]}$, 进而造成岛 弧火山活动的加强.

快速成冰过程显示剧烈的环境变化, 对生态系统造 成巨大的冲击. 相关的科学问题值得进一步研究: 快速成 冰过程的控制因素是什么? 不同生态系统对快速成冰过程 的响应如何? 成冰过程与火山活动的协同作用及其对生物 大灭绝的贡献有多大? 这些问题涉及地球内部与表生环境 的相互作用, 有助于理解宜居星球的形成过程.

\section{参考文献}

1 Harper D A T, Hammarlund E U, Rasmussen C M. End Ordovician extinctions: A coincidence of causes. Gondwana Res, 2014, 25: $1294-1307$

2 Sheehan P M. The Late Ordovician mass extinction. Annu Rev Earth Planet Sci, 2001, 29: 331-364

3 Wang G X, Zhan R B, Percival I G. The end-Ordovician mass extinction: A single-pulse event? Earth-Sci Rev, 2019, 192: 15-33

4 Courtillot V, Olson P. Mantle plumes link magnetic superchrons to phanerozoic mass depletion events. Earth Planet Sci Lett, 2007, 260: 495-504

5 Finnegan S, Heim N A, Peters S E, et al. Climate change and the selective signature of the Late Ordovician mass extinction. Proc Natl Acad Sci USA, 2012, 109: 6829-6834 
$6 \mathrm{Hu}$ Y H, Zhou J B, Song B, et al. SHRIMP zircon U-Pb dating from K-bentonite in the top of Ordovician of Wangjiawan Section, Yichang, Hubei, China. Sci China Ser D-China Sci, 2008, 51: 493-498 [胡艳华，周继涁，宋彪，等. 中国湖北宜昌王家湾剖面奥陶系顶 部斑脱岩 SHRIMP 锆石 U-Pb 定年. 中国科学 D 辑：地球科学, 2008, 38: 72-77]

7 Ling M X, Zhan R B, Wang G X, et al. An extremely brief end Ordovician mass extinction linked to abrupt onset of glaciation. Solid Earth Sci, 2019, 4: 190-198

8 Crowley J W, Katz R F, Huybers P, et al. Glacial cycles drive variations in the production of oceanic crust. Science, 2015, 347: $1237-1240$

9 Huybers P, Langmuir C. Feedback between deglaciation, volcanism, and atmospheric CO . Earth Planet Sci Lett, 2009, 286: 479-491

$10 \mathrm{Hu}$ Y H, Sun W D, Ding X, et al. Volcanic event at the Ordovician-Silurian boundary: The message from K-bentonite of Yangtze Block (in Chinese). Acta Petrol Sin, 2009, 25: 3298-3308 [胡艳华, 孙卫东, 丁兴, 等. 奥陶纪-志留纪边界附近火山活动记录: 来华南周缘 钾质斑脱岩的信息. 岩石学报, 2009, 25: 3298-3308]

11 Sun W D. The Magma Engine and the driving force of plate tectonics (in Chinese). Chin Sci Bull, 2019, 64: 2988-3006 [孙卫东. 岩浆引 擎与板块运动驱动力. 科学通报, 2019, 64: 2988-3006] 